\title{
Dynamics and Control of a Free-Piston Diesel Engine
}

\author{
Tor A. Johansen*1, Olav Egeland*, Erling Aa. Johannessen ${ }^{* * 2}$ and Rolf Kvamsdal** \\ * Department of Engineering Cybernetics, \\ Norwegian University of Science and Technology, \\ N-7491 Trondheim, Norway. \\ ** Kværner ASA, Technology Development \\ Postboks 169, N-1325 Lysaker, Norway.
}

\begin{abstract}
Free-piston diesel engines are characterized by freely moving pistons without any crankshaft or camshaft connected to the pistons. This allows a compact and efficient engine design, but requires automatic control of the piston motion. This paper present a dynamic mathematical model of a free-piston diesel engine, and a control oriented dynamic analysis leading to a piston motion control structure. Experimental results using a full scale test cylinder are included and show feasibility of the suggested control approach.
\end{abstract}

\section{Introduction}

The free-piston diesel engine concept was developed by Pescara, and engines of various size were manufactured between 1930-1960 by GM, Ford, Renault, Junker, Sigma and others, see (Moiroux 1958, Klotch 1959, Underwood 1957) and the more recent review (Achten 1994). The basic idea of the free-piston diesel engine is to avoid the crankshaft in the traditional diesel engine and to use a power turbine instead to convert energy from the exhaust gas. In addition, the camshaft is replaced by some other mechanism to actuate the valves. Hence, the pistons move freely in the cylinders, mainly influenced by pressure forces. The main advantage of the free-piston diesel engine compared to a traditional diesel engine is that there are few moving parts, making the engine compact and mechanically simple. Furthermore, the two-stroke free piston engine has high thermal efficiency. This is in particular true at part load where one can take advantage of the additional degrees of freedom provided by a freely moving piston and let the positions of the bottom and top dead centers depend on the load in order to optimize efficiency and emissions. Thus, the free-piston diesel engine combines the low weight and compactness of gas turbines with the low fuel consumption of diesel engines. This was the motivation

\footnotetext{
${ }^{1}$ Corresponding author. Email: Tor.Arne.Johansen@itk.ntnu.no

${ }^{2}$ Present address: Rolls-Royce Marine AS, P.O. Box 924 , N-5808 Bergen, Norway
} 
for Kværner ASA, who developed a modern two-stroke high-speed free-piston diesel engine concept aimed at marine applications as an alternative to both gas turbines and traditional diesel engines. The net shaft output of an 8 cylinder engine is about $8 \mathrm{MW}$ with a thermal efficiency of about $50 \%$ (from fuel to shaft) at full load.

Recent theoretical and experimental investigations on some variants of the free piston engine concept are reported in (Hu and Hibi 1990, Hu and Hibi 1991, Goldsborough and Blarigan 1999, Goertz and Peng 2000, Tikkanen and Vilenius 1999, Larmi et al. 2001, Achten et al. 2000), mainly using hydraulic energy conversion, aiming for smaller scale applications. The contribution of the present paper is that it presents a control-oriented dynamic model, energy-based analysis and control structure design, and experimental results on a full scale test cylinder, see also the preliminary paper (Johansen et al. 2001). The companion paper (Johansen et al. 2002) focuses on the details of the engine control system, emphasizing valve and injector timing, piston motion parameter estimation, signal processing, low-level control and its implementation.

\section{Free-piston diesel engine operating principles}

Figure 1 provides a sketch of a free-piston diesel engine. The air cushion chamber is indicated by subscript $a$, the combustion chamber by $b$, and the compression chamber by $c$. There is an interstage receiver chamber, denoted $r$, and common intake and exhaust manifolds denoted $i$ and $e$, respectively. The index $p$ refers to a utility air reservoir, and the ambient stagnation state is denoted 0.

The two-stroke diesel cycle, seen from the intake manifold to the exhaust manifold, can be described as follows (see also (Heywood 1988)). Compression: The air in the combustion chamber is compressed when the piston moves from its bottom dead center towards its top dead center. Combustion: Near the top dead center, the temperature of the compressed air is high enough for auto-ignition, and diesel is injected at high pressure into the combustion chamber through a nozzle, and combustion of the diesel fuel results. Air intake: During this upwards motion of the piston, fresh air is sucked into the compressor chamber from the intake manifold through passive suction valves. Expansion: The high pressure in the combustion chamber makes the piston move downwards and the exhaust gas expands. Scavenging: During this downwards motion, the combustion finishes and the actively controlled exhaust valves opens at high pressure to deliver high-energy exhaust gas to the exhaust manifold and turbines. When the piston is at some distance from its nominal bottom dead center, scavenging ports are uncovered and the fresh air in the interstage receiver flushes the combustion chamber and replaces the exhaust gas. Compression in piston compressor: The interstage receiver is at the same time filled by high-pressure air that is compressed in the piston compressor chamber during the downwards motion of 
the piston and delivered through some actively controlled valves connecting the piston compressor chamber and interstage receiver. The high pressure in the compressor chamber and air cushion makes the piston move upwards from its bottom dead center and the cycle repeats.

It is clear that this free-piston concept is feasible only if the piston motion can be controlled. The requirements in terms on control accuracy and robustness are high as cycle-to-cycle variations in the motion of the piston at stationary running will introduce undesired mechanical vibrations and disturbances in the intake and exhaust manifold. Furthermore, there are hard constraints on the motion of the piston due to mechanical stop if the pistons hits the top or bottom of the cylinder.

\section{Mathematical Dynamic Model}

In this section we present a mathematical model based on ordinary differential equations describing mass- and energy-balances of the main gas volumes, and a force balance for the piston. For simplicity, we present the basic equations under the assumptions of the gas being ideal and heat losses, gravity and friction are negligible. This is sufficiently accurate for the control design, as friction and gravity forces are typically around two orders of magnitude smaller than pressure forces and heat losses are typically a few percent of the total heat balance. Dot notation is used to denote time differentiation so that $\dot{m}$ is the time derivative of $m$, that is, $\dot{m}=\frac{d}{d t} m$, and not the mass flow as in common in the combustion engine literature. Mass flow from $i$ to $j$ is denoted by $M_{j i}$. The variable $x$ is the piston position, $T$ denotes absolute temperature, $p$ denotes pressure, $V$ is volume, $\rho$ is density and $m$ is mass. The indices follows the notation introduced in Figure 1.

The inner piston diameter corresponding to the air cushion and combustion chamber is $d_{p i}$. The outer part of the piston corresponding to the compressor chamber has diameter $d_{p o}$. The cross section areas for the air cushion,

combustion chamber and compression chamber are $A_{a}=d_{p i}^{2} \pi / 4, A_{b}=d_{p i}^{2} \pi / 4$, and $A_{c}=\left(d_{p o}^{2}-d_{p i}^{2}\right) \pi / 4$, respectively. The volume of the air cushion chamber is $V_{a}=A_{a}\left(x_{0 a}-x\right)$ where $x_{0 a}$ is the effective length of the air cushion. The volume of the combustion chamber is $V_{b}=A_{b}\left(x_{0 b}+x\right)$, where $x_{0 b}$ accounts for dead volume at the piston upper stop position, corresponding to mechanical contact with the cylinder top. The volume of the compression chamber is $V_{c}=A_{c}\left(x_{0 c}-x\right)$ where $x_{0 c}$ is the effective length of the compression chamber, including dead volume at the piston lower stop position. The volume of the receiver chamber and exhaust manifolds is $V_{r}$ and $V_{e}$, respectively.

The position $x$ increases when the piston moves downwards, cf. Figure 2. The piston is said to be at the top dead center (TDC) when $x$ is at its minimum for the actual cycle. The velocity at this point satisfies $\dot{x}=0$. The 
position at TDC for the actual cycle is defined to be $x_{T D C}$. Likewise, the bottom dead center (BDC) is denoted $x_{B D C}$. This position is reached when $x$ is at its maximum for the actual cycle. Some additional geometric parameters are defined in Figure 2. The equation of motion for the piston (with mass $m$ ) is:

$$
m \ddot{x}=-p_{a} A_{a}-p_{c} A_{c}+p_{0} A_{c}+p_{b} A_{b}
$$

Conservation of mass in the air cushion chamber:

$$
\dot{m}_{a}=M_{a p}
$$

For use in the energy balances, the mass flows are expressed as $M_{a p}=M_{a p}^{\mathrm{in}}-M_{a 0}^{\text {out }}$ where superscript "in" refers to flow in to the air cushion, while superscript "out" refers to flow out. The variables $M_{a p}^{\text {in }}$ and $M_{a 0}^{\text {out }}$ are both non-negative. Conservation of mass in combustion chamber:

$$
\dot{m}_{b}=M_{b r}-M_{e b}
$$

where the mass flows are $M_{b r}=M_{b r}^{\text {in }}-M_{b r}^{\text {out }}$ and $M_{e b}=M_{e b}^{\text {out }}-M_{e b}^{\text {in }}$, where $M_{b r}^{\text {in }}, M_{b r}^{\text {out }}, M_{e b}^{\text {out }}$ and $M_{e b}^{\text {in }}$ are non-negative, and the superscript "in" refers to mass flow into the combustion chamber, while the superscript "out" refers to mass flow out of the combustion chamber. Conservation of mass in compression chamber:

$$
\dot{m}_{c}=M_{c i}-M_{r c}
$$

The mass flows are $M_{r c}=M_{r c}^{\text {out }}-M_{r c}^{\text {in }}$ where $M_{r c}^{\text {out }}$ and $M_{r c}^{\text {in }}$ are non-negative. The superscript "in" refers to mass flow into the compressor chamber, while the superscript "out" refers to mass flow out of the compressor chamber. Conservation of mass in receiver chamber and exhaust manifold:

$$
\begin{aligned}
& \dot{m}_{r}=M_{r c}-M_{b r} \\
& \dot{m}_{e}=-M_{t e}+\sum M_{e b}
\end{aligned}
$$

where the sum is over multiple cylinders.

The energy balances for the air cushion, compressor, combustion, interstage receiver chambers and exhaust manifold can be derived (see (Moran and Shapiro 1993))

$$
\begin{aligned}
\dot{T}_{b}= & \frac{M_{b r}^{\mathrm{in}}}{m_{b}} \kappa T_{r}+\frac{M_{e b}^{\mathrm{in}}}{m_{b}} \kappa T_{e}-\frac{M_{b r}^{\mathrm{in}}+M_{e b}^{\mathrm{in}}+\left(M_{b r}^{\text {out }}+M_{e b}^{\text {out }}\right)(\kappa-1)}{m_{b}} T_{b} \\
& -\frac{1}{m_{b} c_{v}} A_{b} p_{b} \dot{x}+\frac{h_{d i} M_{d i}}{m_{b} c_{v}} \\
\dot{T}_{a}= & \frac{M_{a p}^{\mathrm{in}}}{m_{a}}\left(\kappa T_{p}-T_{a}\right)-\frac{M_{a p}^{\text {out }}(\kappa-1)}{m_{a}} T_{a}+\frac{1}{m_{a} c_{v}} A_{a} p_{a} \dot{x} \\
\dot{T}_{c}= & \frac{M_{c i}}{m_{c}}\left(\kappa T_{i}-T_{c}\right)-\frac{M_{r c}^{\text {in }}+M_{r c}^{\text {out }}(\kappa-1)}{m_{c}} T_{c}+\frac{M_{r c}^{\text {in }}}{m_{c}} \kappa T_{r}+\frac{1}{m_{c} c_{v}} A_{c}\left(p_{c}-p_{0}\right) \dot{x}
\end{aligned}
$$




$$
\begin{aligned}
& \dot{T}_{r}=\frac{M_{r c}^{\text {out }}}{m_{r}} \kappa T_{c}+\frac{M_{b r}^{\text {out }}}{m_{r}} \kappa T_{b}-\frac{M_{r c}^{\text {out }}+M_{b r}^{\text {out }}+\left(M_{r c}^{\text {in }}+M_{b r}^{\text {in }}\right)(\kappa-1)}{m_{r}} T_{r} \\
& \dot{T}_{e}=-\frac{M_{t e}}{m_{e}}(\kappa-1) T_{e}+\sum\left(\frac{M_{e b}^{\text {out }}}{m_{e}}\left(\kappa T_{b}-T_{e}\right)-\frac{M_{e b}^{\text {in }}}{m_{e}}(\kappa-1) T_{e}\right)
\end{aligned}
$$

where $\kappa=c_{p} / c_{v}$, and the sum is over multiple cylinders. Mass flows through valves and ports are modelled as isentropic flow through nozzles (Heywood 1988). Let the inlet stagnation pressure be denoted $p_{1}$ and the nozzle exit pressure, which is assumed to be equal to the throat pressure, be denoted by $p_{2}$. Then the critical exit pressure corresponding to choked flow is

$$
p_{C}=\left(\frac{2}{\kappa+1}\right)^{\frac{\kappa}{\kappa-1}} p_{1}
$$

The mass flow through the valve is

$$
M\left(A, \rho_{1}, p_{1}, p_{2}, \kappa\right)= \begin{cases}A \sqrt{\frac{2 \kappa}{\kappa-1} p_{1} \rho_{1}\left[\left(\frac{p_{2}}{p_{1}}\right)^{\frac{2}{\kappa}}-\left(\frac{p_{2}}{p_{1}}\right)^{\frac{\kappa+1}{\kappa}}\right]} & \text { when } p_{2}>p_{C} \\ A \sqrt{\kappa p_{1} \rho_{1}\left(\frac{2}{\kappa+1}\right)^{\frac{\kappa+1}{\kappa-1}}} & \text { when } p_{2} \leq p_{C}\end{cases}
$$

The mass flow through the valves from the compressor chambers to the receiver chamber are also nonnegative and given by

$$
\begin{aligned}
& M_{r c}^{\text {out }}= \begin{cases}M\left(A_{r c}, \rho_{c}, p_{c}, p_{r}, \kappa\right) & \text { when } p_{r} \leq p_{c} \\
0 & \text { otherwise }\end{cases} \\
& M_{r c}^{\text {in }}= \begin{cases}M\left(A_{r c}, \rho_{r}, p_{r}, p_{c}, \kappa\right) & \text { when } p_{r} \geq p_{c} \\
0 & \text { otherwise }\end{cases}
\end{aligned}
$$

The mass flows into the combustion chambers from the interstage receiver are

$$
M_{b r}^{\text {in }}= \begin{cases}M\left(A_{b r}, \rho_{r}, p_{r}, p_{b}, \kappa\right) & \text { when } x \geq x_{I V O} \text { and } p_{r} \geq p_{b} \\ 0 & \text { otherwise }\end{cases}
$$

The mass flows out of the combustion chambers into the interstage receiver are

$$
M_{b r}^{\text {out }}= \begin{cases}M\left(A_{b r}, \rho_{b}, p_{b}, p_{r}, \kappa\right) & \text { when } x \geq x_{I V O} \text { and } p_{r} \leq p_{b} \\ 0 & \text { otherwise }\end{cases}
$$

The combustion chamber and interstage receiver are connected by ports that are gradually uncovered when the piston position is $x>x_{I V O}=x_{I V C}$. Thus, $A_{b r}=d_{b r} \max \left(0, \min \left(x-x_{B D C}^{n o m}, x-x_{I V O}\right)\right)$. The flow out of the combustion chamber through the exhaust valves is

$$
M_{e b, n}^{\text {out }}= \begin{cases}M\left(A_{e b}, \rho_{b}, p_{b}, p_{e}, \kappa\right) & \text { when }\left(\left(x \geq x_{E V O} \text { and } \dot{x}>0\right) \text { or }\left(x \geq x_{E V C} \text { and } \dot{x}<0\right)\right) \text { and } p_{b} \geq p_{e} \\ 0 & \text { otherwise }\end{cases}
$$


while the flows into the combustion chambers through the exhaust valves are

$$
M_{e b}^{\mathrm{in}}= \begin{cases}M\left(A_{e b}, \rho_{e}, p_{e}, p_{b}, \kappa\right) & \text { when }\left(\left(x \geq x_{E V O} \text { and } \dot{x}>0\right) \text { or }\left(x \geq x_{E V C} \text { and } \dot{x}<0\right)\right) \text { and } p_{b} \leq p_{e} \\ 0 & \text { otherwise }\end{cases}
$$

The mass flow in or out of each the air cushion is controlled by a three-level signal: No flow $(u=0)$, flow into the air cushion $(u=1)$, or flow out of the air cushion to atmospheric pressure $(u=-1)$.

$$
\begin{aligned}
M_{a 0}^{\text {out }} & = \begin{cases}M\left(A_{a 0}, \rho_{a}, p_{a}, p_{0}, \kappa\right) & \text { when } u=-1, \text { and } p_{a} \geq p_{0} \\
0 & \text { otherwise }\end{cases} \\
M_{a p}^{\text {in }} & = \begin{cases}M\left(A_{a p}, \rho_{p}, p_{p}, p_{a}, \kappa\right) & \text { when } u=1, \text { and }, p_{p} \geq p_{a} \\
0 & \text { otherwise }\end{cases}
\end{aligned}
$$

The mass flow through the passive compressor suction valves are nonnegative and given by

$$
M_{c i}=M\left(A_{c i}, \rho_{i}, p_{i}, p_{c}, \kappa\right)
$$

where $A_{c i}=\min \left(A_{c i}^{\max }, d_{c i} \xi\right)$ is the effective area of flow, $A_{c i}^{\max }$ the maximum value, and $\xi$ is the valve position. The equation of motion for the valves is:

$$
m_{c i} \ddot{\xi}=-D_{c i} \dot{\xi}+A_{c i}^{\max }\left(p_{i}-p_{c}\right)-k_{c i}\left(\xi^{0}+\xi\right)
$$

where $\xi$ is the valve position, $D_{c i}$ is the damping, $d_{c i}$ is the valve outer circumference, $k_{c i}$ is the spring constant, $m_{c i}$ is the valve mass and $\xi^{0}$ is the position offset. In all actuated valves the model takes into account the actuator delays. The diesel heat release $M_{d i} h_{d i}$ is initiated at some piston position $x_{C S}$, and assumed to evolve according to some time-varying function that depends on the diesel pressure and injected amount, see e.g. (Heywood 1988, Borman and Ragland 1998). At full load the thermal efficiency is about $50 \%$ (from fuel to shaft), mass flow through the cylinder is about $1.75 \mathrm{~kg} / \mathrm{s}$ per cylinder, stroke length about $189 \mathrm{~mm}$, intake pressure (from turbo compressor) about 3.25 bar, exhaust pressure about 20 bar, exhaust temperature about 1050 $\mathrm{K}$, and piston frequency about $1800 \mathrm{rpm}$. The mathematical model was tuned and verified against experimental data, (Johansen et al. 2001). In particular, theoretical valve areas were reduced somewhat to account for losses.

\section{Model Analysis}

The purpose of this section is to analyze how the top dead center (TDC) position $x_{T D C}$ and bottom dead center (BDC) position $x_{B D C}$, which we want to control, can be influenced by manipulating the free variables 
we have at hand. The basic control variables available are the signals to the injector, exhaust valves, piston compressor delivery valves, and in/out valves at the air cushion. These are all on/off type of valves that can be commanded open or closed. These control variables offer considerable degrees of freedom, but there are also certain constraints on their usefulness:

- Injected diesel will only combust when the temperature in the cylinder is sufficiently high, i.e. near the TDC. In other words, the timing is more or less fixed, and optimized for efficiency and emissions, but we can command the amount of diesel $m_{d i}$ injected at each cycle with high accuracy to the common rail diesel injection system.

- The freedom in timing of the exhaust valves is also constrained by the requirement that they must open sufficiently late in the expansion stroke such that combustion is finished, and they must open sufficiently early in the expansion stroke such that the exhaust pressure is sufficiently low to prevent back-flow when the scavenging ports are uncovered. Furthermore, the exhaust valves must close sufficiently early in the compression stroke to ensure sufficient compression for auto-ignition.

- The compressor delivery valves should essentially be timed to simulate passive valves that open when the differential pressure is positive. Any other timing would lead to unnecessary losses and is therefore undesirable to use during normal operation.

- The air cushion valves have essential full flexibility to add or remove air from the air cushion within the rate constraints on the mass flow due to the size of the valves. In order to minimize the use of utility air, it is however desirable to keep the use of these valve to a minimum and avoid taking air in and out of the air cushion within the same cycle.

From the above discussion, it is clear that the most useful control variables are the air cushion mass $m_{a}$ and the amount of injected diesel per cycle $m_{d i}$. Notice that the reason why $m_{d i}$ is a free variable is that the load of the engine is controlled by the supervisory control control actuating on the turbo-machinery and commanding set-points to the motion control system to achieve the desired exhaust manifold pressure when accelerating or retarding the engine. Notice that $m_{d i}$ can be specified directly to the diesel injection system through a mapping that maps diesel pressure and desired volume of injected diesel to an electronic pulse. The air cushion mass $m_{a}$ is indirectly controllable, and is the set-point to a low level control system which includes an estimator for the air cushion mass, (Johansen et al. 2002).

Here we establish how the steady-state values of $x_{T D C}$ and $x_{B D C}$ can be influenced by $m_{d i}$ and $m_{a}$. In order to relate $m_{d i}$ and $m_{a}$ to $x_{B D C}$ we consider the expansion stroke, i.e. piston motion from TDC to BDC. The 
additional work $\Delta W$ needed to change the stroke length by $\Delta x_{B D C}$ near the BDC is approximately

$$
\Delta W=\left(p_{a}^{B D C} A_{a}+\left(p_{c}^{B D C}-p_{0}\right) A_{c}-p_{b}^{B D C} A_{b}\right) \Delta x_{B D C}
$$

when assuming $\Delta x_{B D C}$ is small. Furthermore, one may approximate $p_{c}^{B D C} \approx p_{b}^{B D C} \approx p_{r}$ if the pressure loss over the valves are negligible. This additional work may be produced by additional fuel input, so

$$
\Delta m_{d i} h_{d i}=\left(m_{a} A_{a} R \bar{T}_{a} \frac{\bar{V}_{a}^{\kappa-1}}{\left(V_{a}^{B D C}\right)^{\kappa}}+\left(p_{r}-p_{0}\right) A_{c}-p_{r} A_{b}\right) \Delta x_{B D C}
$$

where we have used the ideal gas law and isentropic compression relations

$$
p_{a}^{B D C}=m_{a} R \bar{T}_{a} \frac{\bar{V}_{a}^{\kappa-1}}{\left(V_{a}^{B D C}\right)^{\kappa}}
$$

where $\bar{T}_{a}$ and $\bar{V}_{a}$ are the cycle-averaged volume and temperature of the air cushion. Hence, in order to change the position of the BDC by $\Delta x_{B D C}$ from one cycle to the next while keeping $m_{a}$ constant, one must add $\Delta m_{d i}$ defined by (25) to the mass of injected fuel.

The differential influence of air cushion mass $m_{a}$ and amount of injected fuel $m_{d i}$ on $x_{T D C}$ can be found by considering the compression stroke, i.e. the piston motion from BDC to TDC. The additional work on the piston needed to change the stroke length by a small amount $\Delta x_{T D C}$ near the TDC is approximately

$$
\Delta W=\left(p_{b}^{T D C} A_{b}-\left(p_{c}^{T D C}-p_{0}\right) A_{c}-p_{a}^{T D C} A_{a}\right) \Delta x_{T D C}
$$

Again, one may approximate $p_{c}^{T D C} \approx p_{i}$ and $p_{b}^{T D C} \approx p_{r} r_{d i} \alpha_{d i}$, where $r_{d i}$ is the combustion chamber pressure ratio, and $\alpha_{d i}>1$ is a factor that accounts for the peak pressure increase due to combustion. At idle $\alpha_{d i} \approx 2$ while at full load $\alpha_{d i}$ is only slightly larger than 1 (because of the long injection period at full load only a small fraction of the diesel will be combusted when the peak pressure is reached). The combustion chamber pressure ratio depends on the pressure ratio achieved in the piston compressor such that the total pressure ratio (piston compressor plus combustion chamber) is constant, $r_{d i}=r_{t} p_{i} / p_{r}$, where $r_{t}$ is a constant. Furthermore, using the ideal gas law and isentropic compression relations we get

$$
p_{a}^{T D C}=m_{a} R \bar{T}_{a} \frac{\bar{V}_{a}^{\kappa-1}}{\left(V_{a}^{T D C}\right)^{\kappa}}
$$

The change in work $\Delta W$ may be caused by a change in the internal energy of the air cushion $\Delta U_{a}=c_{v} T^{\prime} \Delta m_{a}$ by changing the amount of air by $\Delta m_{a}$

$$
c_{v} T^{\prime} \Delta m_{a}=\left(p_{i} r_{t} \alpha_{d i} A_{b}-\left(p_{i}-p_{0}\right) A_{c}-m_{a} A_{a} R \bar{T}_{a} \frac{\bar{V}_{a}^{\kappa-1}}{\left(V_{a}^{T D C}\right)^{\kappa}}\right) \Delta x_{T D C}
$$

where $T^{\prime}=T_{a}$ when $\Delta m_{a}<0$ and $T^{\prime}=T_{p}$ when $\Delta m_{a}>0$. 


\section{Control Structure}

The analysis in section 4 is confirmed by simulation of the dynamic model in section 3 . It is evident that the simplest control structure consists of two SISO controllers where $m_{d i}$ is paired with $x_{B D C}$ and $m_{a}$ is paired with $x_{T D C}$. The use of $m_{d i}$ to control the stroke length is similar to the ideas presented in (Tikkanen and Vilenius 1999). Although a $2 \times 2$ MIMO controller might perform better, the experimental results show that the suggested control structure gives adequate performance.

Integral action is required in both control loops because it is essential that the steady-state error is small, and there may be a significant offset in the diesel injection system such that the actual $m_{d i}$ differs from the commanded $m_{d i}^{*}$. Furthermore, there may be a large offset also in the air cushion mass estimator due to a poor guess of the mean temperature such that the actual $m_{a}$ differs from its estimate $\hat{m}_{a}$. The results from model analysis suggest the relationships (25) and (29) that can be rewritten in the form

$$
\begin{aligned}
\Delta m_{d i} & =\left(k_{0}+k_{1} m_{a}+k_{2} p_{r}\right) \Delta x_{B D C} \\
\Delta m_{a} & =\left(g_{0}+g_{1} m_{a}+g_{2} p_{r}+g_{3} p_{i}\right) \Delta x_{T D C}
\end{aligned}
$$

where $k_{0}, k_{1}, k_{2}, g_{0}, g_{1}, g_{2}$ and $g_{3}$ are constants. Obviously, this suggests some controller nonlinearities. The variables $m_{a}, p_{i}$ and $p_{r}$ are roughly invariant over each cycle and depends mainly on the load of the engine. At idle, $p_{i}=1$ bar and it increases gradually to $p_{i}=3.25$ bar at full load. Moreover, $p_{r}=2$ bar at idle and it increases to $p_{r}=22 \mathrm{bar}$ at full load. Moreover, $m_{a}=16 \mathrm{~g}$ at idle and it increases to about $m_{a}=80 \mathrm{~g}$ at full load. Figure 3 illustrates theoretical dependencies of $x_{T D C}^{*}, x_{B D C}^{*}, m_{a}^{*}, m_{d i}^{*}, p_{r}$ and $p_{i}$ as a function of load. These are computed using standard ideal thermodynamic relationships in order to achieve an exhaust gas temperature that is independent of the load. The curves for $x_{T D C}$ and $x_{B D C}$ are used as load-dependent set-points for the piston motion control system. One may rewrite (30) and (31) as

$$
\begin{aligned}
\Delta m_{d i} & =k(L) \Delta x_{B D C} \\
\Delta m_{a} & =g(L) \Delta x_{T D C}
\end{aligned}
$$

where $L$ is load varying between approximately $5 \%$ at idle to $100 \%$. These are illustrated in Figure 4 . It should be noted that even though the individual terms of (31) are highly nonlinear it is clear that (33) are much less nonlinear as a function of $L$ because the individual terms will dominate at different loads.

The main contribution to the dynamic response is the volume of the interstage receiver chamber. A rough estimate of the dominant time-constant might be found by computing the ratio of the volume $V_{r}$ to the volumetric flow rate $q_{r}=\bar{M}_{r} / \rho_{r}$, where $\bar{M}_{r}$ is the mass flow through the interstage receiver averaged over a cycle. Notice 
that $V_{r}$ is constant, while $q_{r}$ may show some variation between idle and full load. However, this variation is negligible since the air-to-fuel ratio does not vary too much (although it is somewhat higher at idle than at full load). Hence, $\tau=V_{r} / q_{r}$ can be considered to be load independent. In fact, full scale experimental results show that adequate performance can be achieved with linear controllers (PI control of the air cushion and PID control of the diesel injection) over the full operating range, even though a nonlinear diesel controller where the gain is scheduled on the load of the engine consistent with (32) might give consistently better performance over the full operating range.

\section{Experimental Results}

A single cylinder of the diesel combustion unit has been tested in full scale, see (Johansen et al. 2002) for a more detailed description of the experimental setup. The single cylinder test unit working point is defined by the charge air pressure before the compressor chamber and the exhaust manifold pressure. The charge air pressure is controlled by a valve on the inlet pipe, after the intercooler. The exhaust pressure is controlled by a valve on the exhaust manifold outlet pipe. By adjusting these pressures different compressor and turbine maps may be simulated, cf. Figure 1. Results of transient running of the engine are shown in Figure 5. Initially, the piston is at rest at the PBS position and the pressure throughout the engine is atmospheric. The engine starts at $t \approx 2.0 \mathrm{~s}$ by injecting the sufficient amount of air $m_{a}$ into the air cushion to generate the pressure required to make the piston move to the desired TDC position. At the TDC the required amount of diesel $m_{d i}$ is injected to take the piston to the desired BDC position. At this point, the piston motion controller takes over, and commands $m_{a}$ and $m_{d i}$ for each cycle. The engine load is increased from idle to about $40 \%$ during this time interval, by choking the exhaust gas flow. We observe from the curves that the piston motion control system operates satisfactory with variability of the dead center position of about $1 \mathrm{~mm}$ after the initial transient. Somewhat larger variability must be accepted at the startup.

\section{References}

Achten, P. A. J. (1994). A review of free piston engine concepts. In: SAE Paper 941776.

Achten, P. A. J., J. P. J. Van Den Oeven, J. Potma and G. E. M. Vael (2000). Horsepower with brains: the design of the CHIRON free piston engine. In: SAE Technical paper 012545.

Borman, G. and K. Ragland (1998). Combustion Engineering. McGraw-Hill. 
Goertz, M. and L. X. Peng (2000). Free piston engine - its application and optimization. In: SAE Technical paper 000996.

Goldsborough, S. S. and P. V. Blarigan (1999). A numerical study of a free piston internal combustion engine operating on homogeneous charge compression ignition combustion. In: SAE Technical paper 990619.

Heywood, J. B. (1988). Internal Combustion Engine Fundamentals. McGraw-Hill.

Hu, Y. and A. Hibi (1990). Hydraulic free piston internal combustion engine (experimental investiagation of the fundamental test apparatus with opposed piston). Trans. Japan Soc. Mech. Engr., Series B 56, 3167-3172.

$\mathrm{Hu}$, Y. and A. Hibi (1991). Hydraulic free piston internal combustion engine (theoretical investiagation of the opposed piston type). Trans. Japan Soc. Mech. Engr., Series B 57, 762-767.

Johansen, T. A., O. Egeland, E. A. Johannessen and R. Kvamsdal (2001). Free-piston diesel engine dynamics and control. In: Proc. American Control Conference, Arlington, Va.

Johansen, T. A., O. Egeland, E. A. Johannessen and R. Kvamsdal (2002). Free-piston diesel engine timing and control - towards electronic cam- and crankshaft. IEEE Trans. Control Systems Technology 10, 177-190.

Klotch, P. (1959). Ford free-piston engine development. In: SAE Paper 590045.

Larmi, M., S. Isaksson, S. Tikkanen and M. Lammila (2001). Performance simulation of a compression ignition free piston engine. In: SAE Technical paper 010280.

Moiroux, A. F. (1958). Free-piston engine possibilities. In: ASME Oil and Gas Power Conference. pp. 58OGP-7.

Moran, M. J. and H. N. Shapiro (1993). Fundamentals of Engineering Thermodynamics, 2nd edition. Wiley.

Tikkanen, S. and M. Vilenius (1999). Hydraulic free piston engine - challenge for control. In: Proc. European Control Conference, Karlsruhe.

Underwood, A. F. (1957). The GMR 4-4 Hyprex engine - a concept of the free-piston engine for automotive use. In: SAE Paper 570032. 


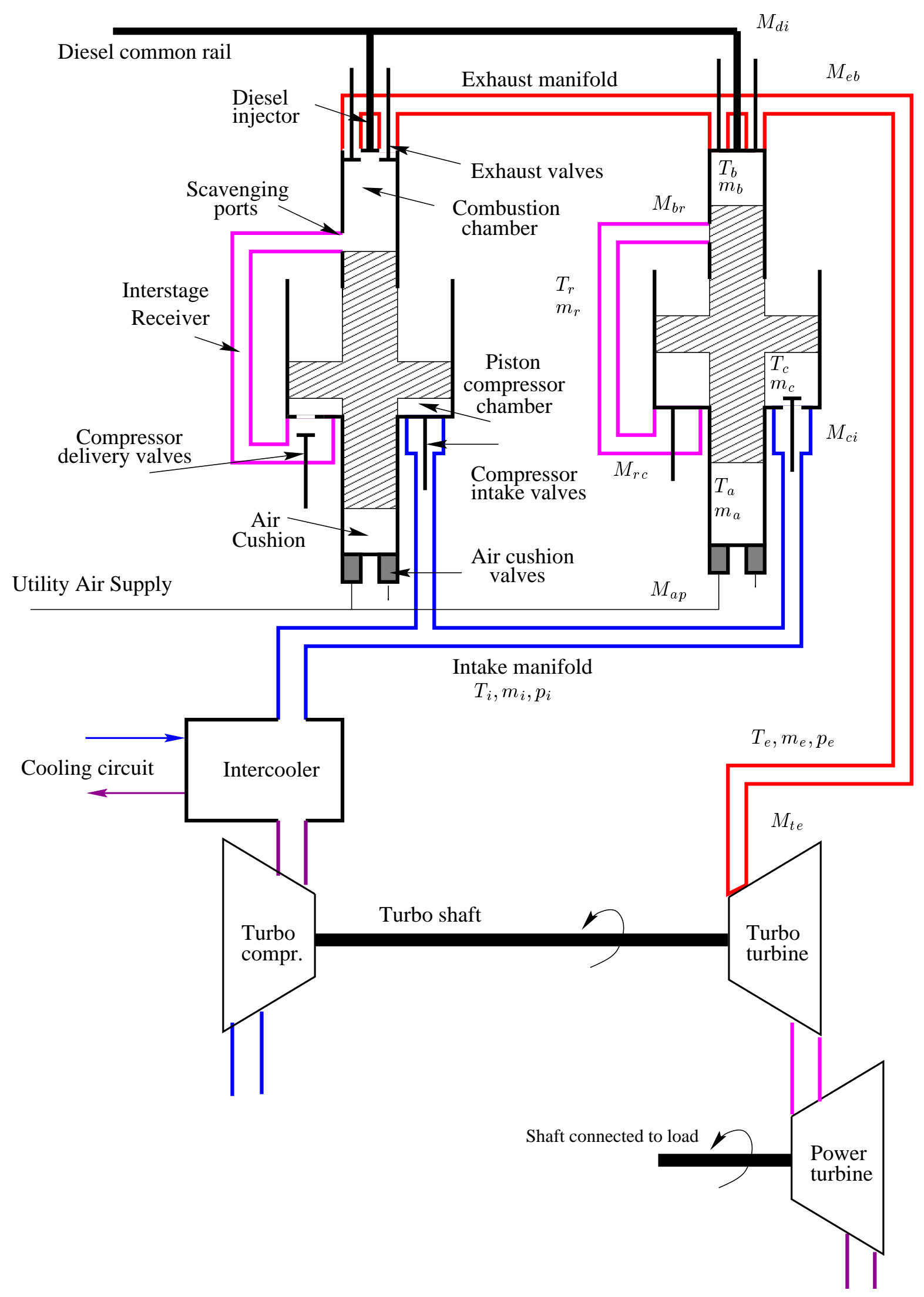

Figure 1: Sketch of multi-cylinder free-piston engine, indicating mass and energy flows. 


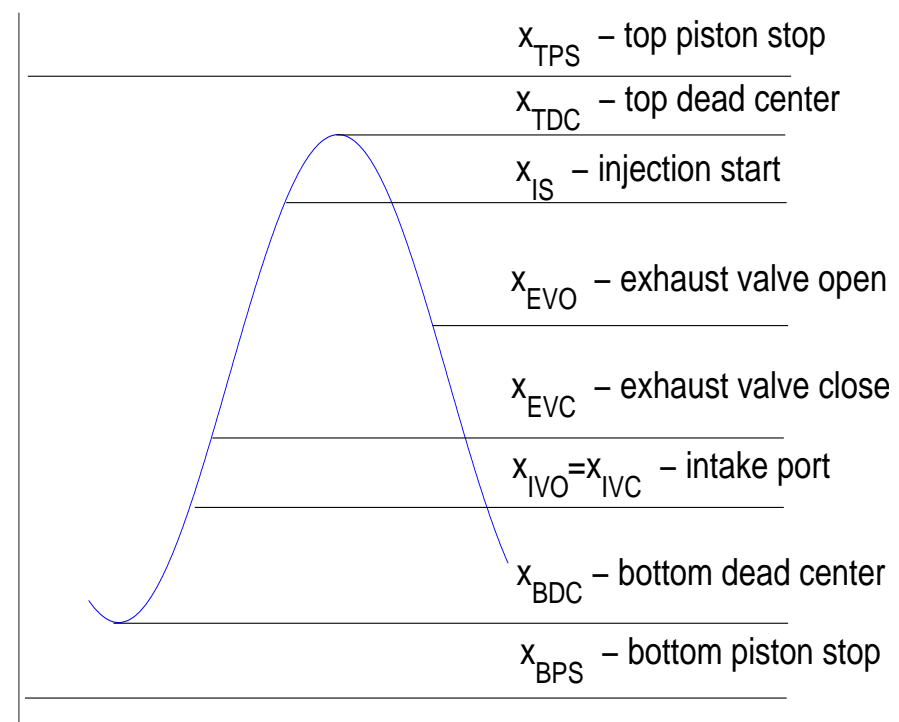

Figure 2: Geometric cycle parameters. 

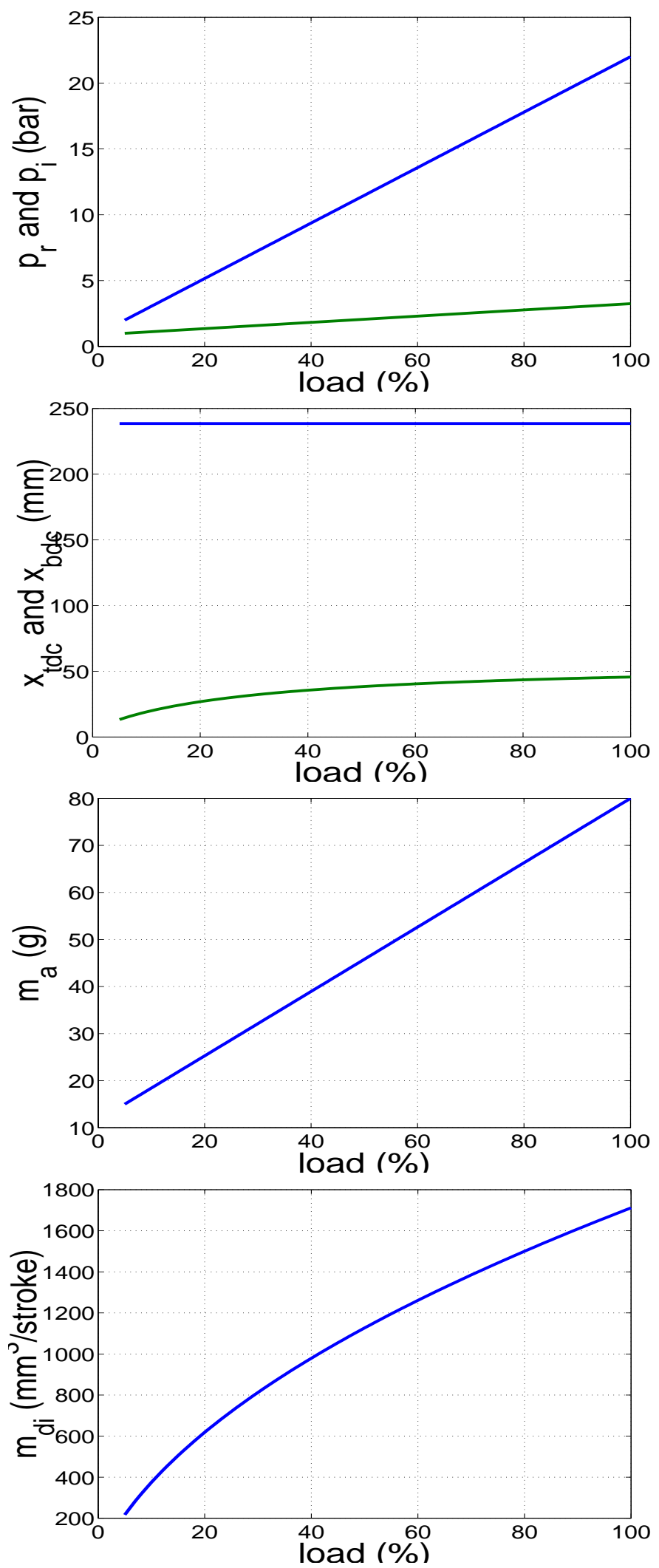

Figure 3: Main engine parameters as a function of load (theoretical calculations). 

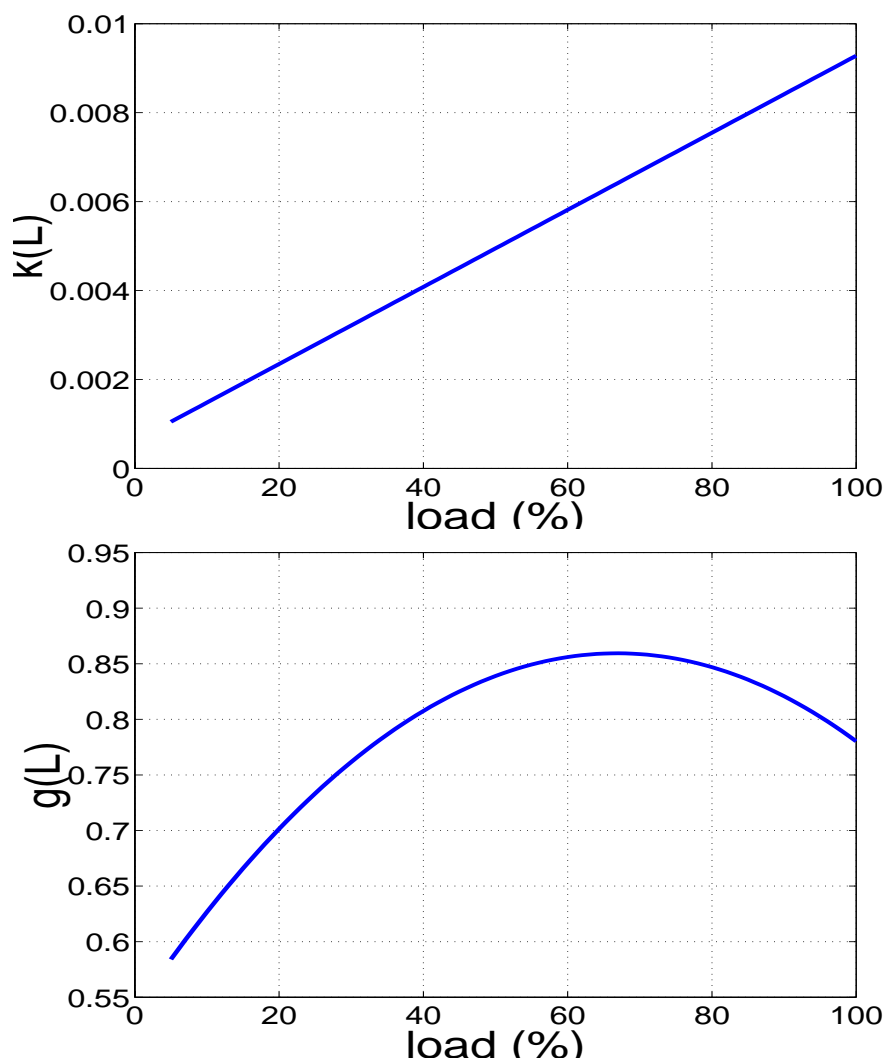

Figure 4: Nonlinear gain as a function of load. 

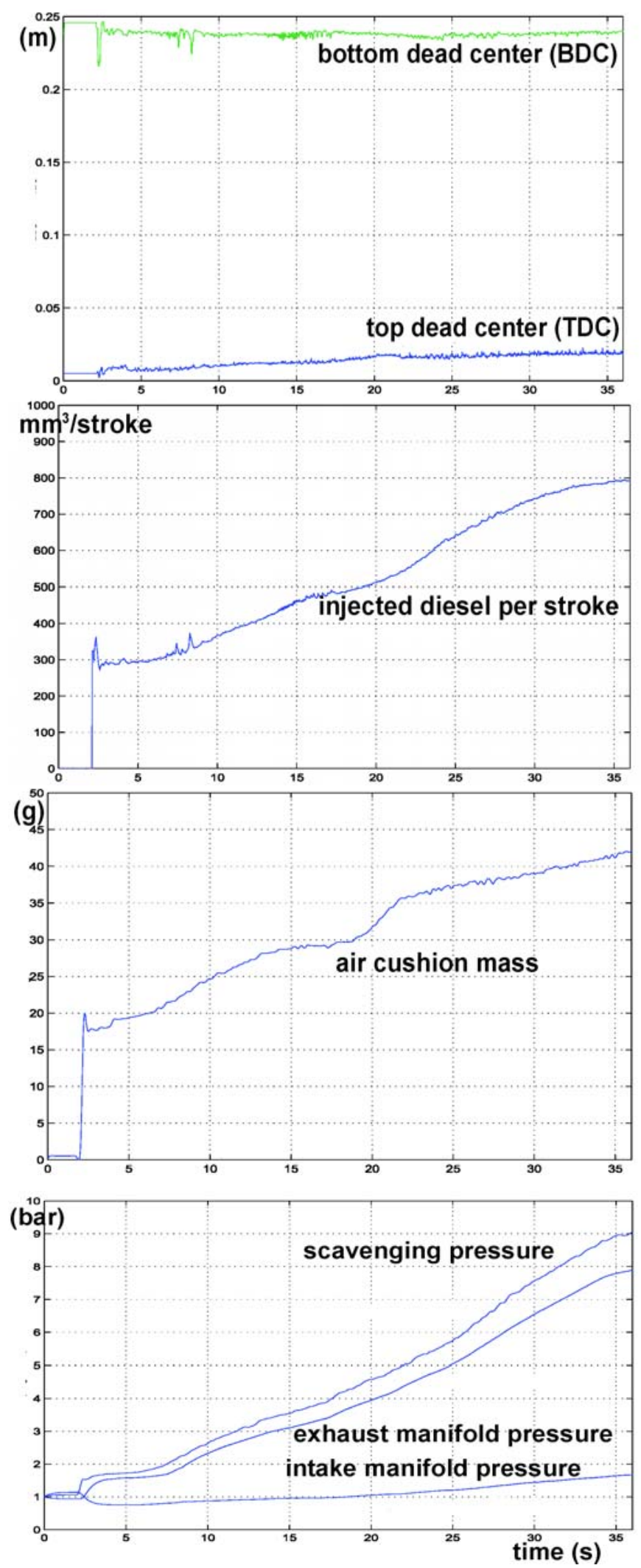

Figure 5: Piston motion ( $x_{B D C}$ and $x_{T D C}$ positions) and control input $m_{d i}$ and $m_{a}$ when the load of the engine is increased from idle to about $40 \%$ using a throttle in the exhaust pipe. Curves for the exhaust manifold pressure $p_{e}$, scavenging pressure $p_{r}$ and intake manifold pressure $p_{i}$ are also shown. 